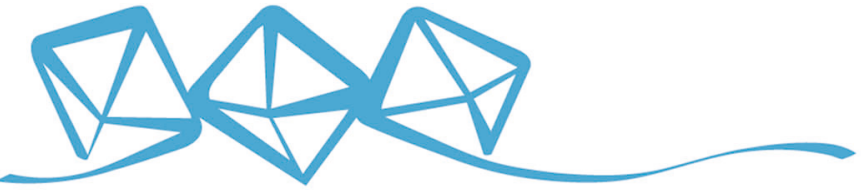 COMMUNICATIONS MATERIALS
}

ARTICLE

Check for updates https://doi.org/10.1038/s43246-020-0045-y

OPEN

\section{Catalysis-free transformation of non-graphitising carbons into highly crystalline graphite}

Jason L. Fogg ${ }^{1}$, Kate J. Putman', Tianyi Zhang ${ }^{2}$, Yu Lei $^{2}$, Mauricio Terrones ${ }^{2,3}$, Peter J. F. Harris ${ }^{4}{ }^{4}$, Nigel A. Marks ${ }^{1} \&$ Irene Suarez-Martinez ${ }^{1 凶}$

High-purity graphite is a sought-after material for lithium-ion batteries and graphene production. Most organic materials do not graphitise upon heating unless a metal catalyst is present. The catalyst becomes embedded in the graphite and is difficult to remove. Here, we present a catalysis-free technique capable of producing highly crystalline graphite from materials generally considered incapable of this transformation. Using the furnace inside an Atomic Absorption Spectrometer, we perform repeated high-temperature pulsing of polyvinylidene chloride followed by analysis with Raman, X-ray diffraction and transmission electron microscopy. Unexpectedly, $\sim 90 \%$ of the sample transforms into highly ordered graphite with very few defects. A combustion route is proposed in which oxygen attacks the structural units that inhibit graphitisation. We apply the same approach to cellulose and obtain ten times more ordered material than conventional furnaces, confirming that polyvinylidene chloride is not an isolated case. Potentially, this method could be used to synthesise graphite from any organic material, including waste sources such as biomass.

\footnotetext{
${ }^{1}$ Department of Physics and Astronomy, Curtin University, Perth, WA, Australia. ${ }^{2}$ Department of Materials Science and Engineering, The Pennsylvania State University, University Park, PA 16802, USA. ${ }^{3}$ Research Initiative for Supra-Materials, Shinshu University, Nagano City, Nagano Prefecture 380-8553, Japan. ${ }^{4}$ Electron Microscopy Laboratory, Department of Chemistry, J.J. Thomson Building, University of Reading, Whiteknights, Reading RG6 6AF, UK.

凶email: I.Suarez-Martinez@curtin.edu.au
} 
G raphite is a naturally occurring mineral, but deposits are rarely found at sufficient quality $(99.95 \%$ carbon) for battery applications and graphene production. Purification uses aggressive acids and generates a harmful waste stream ${ }^{1}$. Alternatively, synthetic graphite can be produced via hightemperature heat treatment of petroleum coke or coal tar' ${ }^{2}$. Graphitisation was first investigated in the 1950s by Rosalind Franklin who coined the terms "graphitising" and "non-graphitising" 3 to categorise carbonaceous precursors; graphitising carbons are a select group of materials that convert to graphite upon heating above $1700^{\circ} \mathrm{C}$, whereas the majority of carbonaceous materials are non-graphitising and show no homogenous order even when heated as high as $3000^{\circ} \mathrm{C}^{3}$.

Franklin's work is the foundation for the International Union of Pure and Applied Chemistry (IUPAC) terminology of carbon solids that divides materials into non-graphitisable and graphitisable carbons ${ }^{4}$. While the IUPAC definition implies a dichotomy, Franklin in fact reports that most non-graphitising carbons contain a few percent of ordered graphitic material. This narrative of a small graphitising phase within a larger non-graphitising structure is not unique, but reports are rare $^{5-8}$ and generally do not refer to each other. Kobayashi et al. ${ }^{5}$ were the first to provide a comprehensive deconvolution of the phases from X-ray diffraction (XRD) data, while Oberlin and Rousseaux ${ }^{6}$ additionally presented a schematic suggesting how graphitising carbon is incorporated into non-graphitising carbons.

In this paper, we perform high-temperature treatments on two classic non-graphitising carbons and demonstrate a process whereby the minority graphite phase increases far beyond the level of a few percent, to the point of graphitising most of the sample ( $90 \%)$. This process is distinct from catalytic graphitisation where metals such as iron and nickel enable the conversion of non-graphitising carbons into graphite at relatively modest temperatures around $2000^{\circ} \mathrm{C}^{9-11}$. The downside of catalysis is that metallic particles are interspersed with the graphite crystals and are difficult to remove. By comparison, our catalyst-free process is capable of producing graphite in a single step. Our method uses the furnace inside an Atomic Absorption Spectrometer (AAS) to conduct consecutive short heating pulses $(<1 \mathrm{~min})$. These fast heating cycles are essential to our process and have not been previously explored owing to the slow heating rate of conventional furnaces, which operate on a timescale of hours.

\section{Results}

Polyvinylidene chloride (PVDC) is a textbook non-graphitising carbon $^{12}$, composed of a repeating monomer of hydrogen, chlorine and carbon (Fig. 1a). After slow carbonisation in argon $\left(4^{\circ} \mathrm{C} / \mathrm{min}\right.$ up to $1000^{\circ} \mathrm{C}$ ), all of the chlorine and most of the hydrogen is removed. This produces a hard and porous solid shown in Fig. 1b, and it is typical of carbonised non-graphitising carbon. The material is then exposed to consecutive high-temperature pulses, in which a rapid heating rate is used to achieve and hold $3000^{\circ} \mathrm{C}$ in a matter of seconds before the sample is cooled under argon flow; typically a few minutes separates each pulse. This unique heating method utilises the small graphite furnace of an AAS, a common piece of equipment in chemistry laboratories. The furnace is a graphite tube $25 \mathrm{~mm}$ long with an inner diameter of $3.6 \mathrm{~mm}$; it is loaded with $\sim 50 \mathrm{mg}$ of carbonised material. After one pulse at $3000^{\circ} \mathrm{C}$, transmission electron microscopy (TEM) images show solely disordered material (Fig. 1c). Ordering develops with further pulsing, forming graphitic planes as shown in Fig. 1d. XRD patterns in Fig. 1e-g further illustrate this transformation. After carbonisation, the material has little order, showing none of the reflections associated with crystalline carbon. After one heating pulse, basic structural units (BSUs) begin to form and $\{002\},\{10\}$ and $\{11\}$ reflections appear in the XRD pattern. After five more pulses, the reflections sharpen considerably and new reflections appear, indicating that Bernal $(\mathrm{AB})$ graphite has formed.

The evolution of ordering is quantified in Fig. 2. Particularly illustrative is the $\{002\}$ reflection associated with the interlayer spacing of graphite. As shown in Fig. 2a, this reflection can be decomposed into three components: a broad peak associated with disordered carbon (D), a sharp peak at $26.0^{\circ}$ associated with randomly turbostratic graphenic carbon $(\mathrm{T})$, and another sharp peak at $26.5^{\circ}$ indicating the presence of graphite $(G)$. After one pulse, the $\{002\}$ reflection is broad and is dominated by the disordered component. The turbostratic peak becomes visible after four pulses with a shoulder evident after five pulses indicating the graphite layering. After six pulses, a dramatic change can be observed in the XRD pattern, where numerous sharp reflections become prominent and the $\{002\}$ reflection shifts entirely to the $26.5^{\circ}$ value associated with crystalline graphite. Significant reflections appear, in particular the $\{101\}$ at $44^{\circ}$ (Fig. 2b) and the $\{112\}$ at $84^{\circ}$. These reflections indicate specific $3 \mathrm{D}$ ordering that only arises from $\mathrm{AB}$ stacking characteristic of Bernal graphite ${ }^{13}$. This indicates that most of the sample has graphitised. This is an astonishing result, as PVDC appears in Franklin's work ${ }^{3}$ and standard carbon reference books ${ }^{2,12}$ as a prototypical nongraphitising material.

To quantify the transformation, we extract quantitative estimates of each phase from the intensity ratio of the components making up the $\{002\}$ reflection. Figure $2 \mathrm{~d}$ shows the turbostratic and graphite components for all our experiments (see full data in Supplementary Table 1). When pulsing up to four times, the ordered components $(\mathrm{T}+\mathrm{G})$ make up a few percent of the sample. Previous reports using conventional furnaces have reported similarly small percentages of ordered components $(<5 \%)$ from high-temperature treatment of non-graphitising carbons ${ }^{3,5,6}$. The logarithmic scale highlights the rapid increase in ordering; after five pulses, the ordered fraction $(T+G)$ is $\sim 10 \%$ while after six pulses the graphite phase completely dominates. The six-pulse sample is around $90 \%$ graphite and has a)

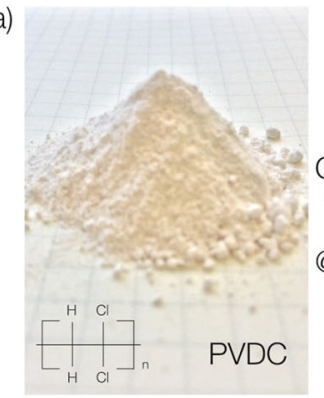

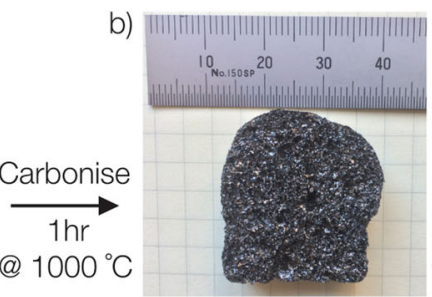

e)

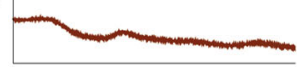

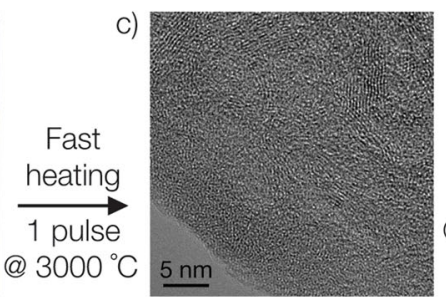

f)

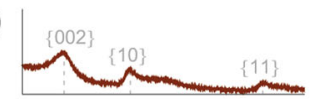

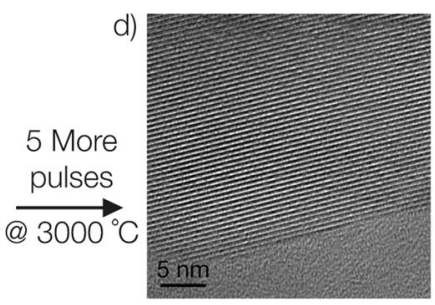

g)

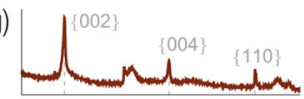

Fig. 1 Outline of the process to transform PVDC into graphite. a Raw PDVC powder, $\mathbf{b}$ carbonised PVDC, $\mathbf{c}$ TEM images of carbonised PVDC treated with one high-temperature pulse, $\mathbf{d}$ TEM after six pulses, e- $\mathbf{g}$ XRD pattern for the samples in $\mathbf{b}-\mathbf{d}$ across a $2 \theta$ range of $10-90^{\circ}$ 
a)

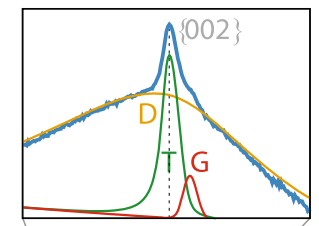

c)
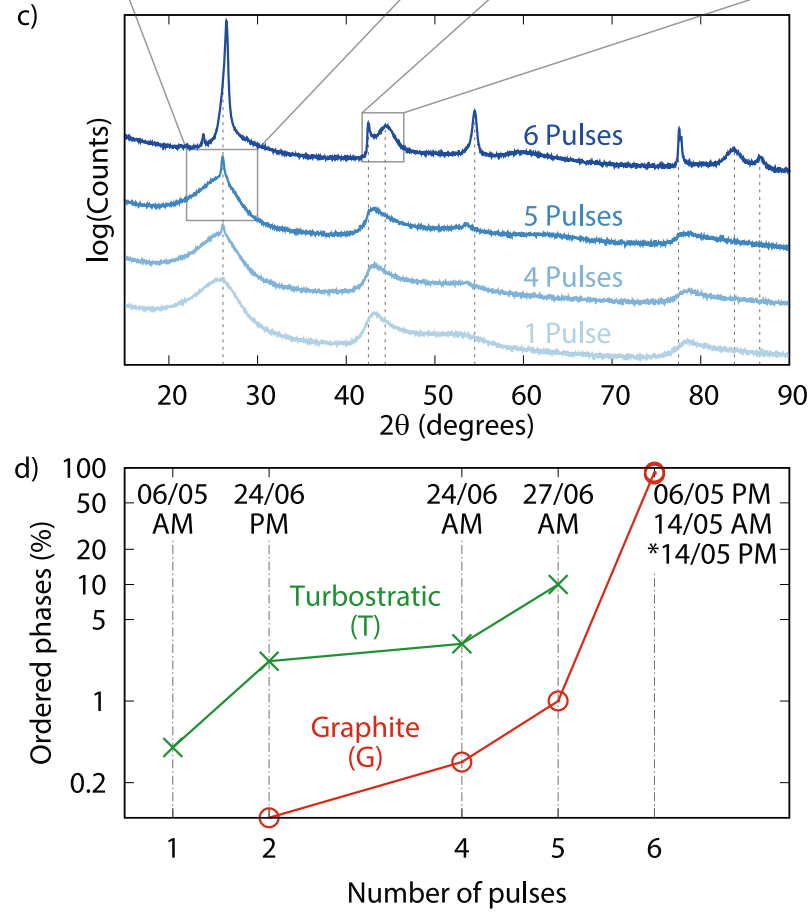

Fig. 2 XRD analysis of PVDC treated with high-temperature pulses.

a Magnification of the $\{002\}$ reflection from the five-pulse sample showing the disordered $(D)$, turbostratic $(T)$ and graphitic $(G)$ components.

b Magnification of the 6-pulse sample demonstrating a high degree of 3D order. c Full XRD patterns for different number of pulses. Dotted lines indicate graphite reflections: from left to right, $\{002\},\{100\},\{101\},\{004\}$, $\{110\},\{112\},\{006\}$. d Percentage of turbostratic and graphite phases as computed from the intensity ratios of the $\{002\}$ reflection. The date at which the heat treatment occurred has been included for each sample. The asterisk indicates a sample produced using a different graphite tube.

no identifiable turbostratic peak, with the remaining material in the disordered phase.

To discount the possibility that contamination is the origin of the graphite component, we performed three checks. First, we confirmed the purity of the as-bought PVDC using energydispersive X-ray spectroscopy. No metallic impurities were detected down to the sensitivity limit of $\sim 0.1 \mathrm{wt} \%$, far below the $15-20 \mathrm{wt} \%$ used to catalyse graphitisation ${ }^{9-11}$. Second, we considered that the graphite may have come from fatigue of the tube furnace. If this were the case, then the amount of graphite would correlate with the number of times a tube is used. The ordering of the time stamps in Fig. 2 d prove that this is not the case. The data are monotonic in the number of pulses, even though the experiments were not performed in chronological order. Finally, the six-pulse sample was replicated three times using two different tubes, and all XRD parameters were essentially identical (see Supplementary Fig. 1).

We use two real-space techniques, Raman spectroscopy and TEM, to characterise carbonised PVDC treated with hightemperature pulses. The results from Raman (Fig. 3a) are consistent with XRD and show that there is an obvious transformation after six pulses. All spectra exhibit the $D, G, D^{\prime}$ and $2 \mathrm{D}$ a)
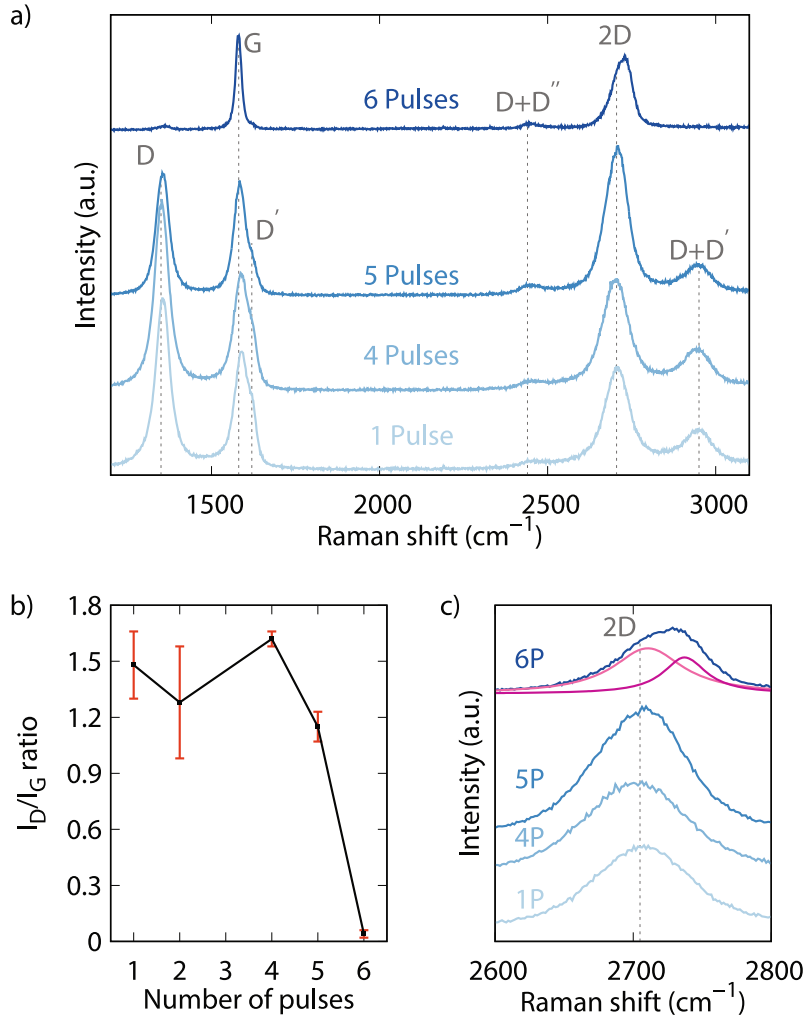

Fig. 3 Raman spectroscopy of PVDC treated with high-temperature pulses. a Raw data for one, four, five and six pulses. $\mathbf{b} / \mathrm{D} / \mathrm{I}_{\mathrm{G}}$ ratios computed from Lorentzian fittings. Error bars indicate $95 \%$ confidence intervals (i.e. twice the standard error of the mean). c Magnification of the 2D band showing the splitting of the peak for the six-pulse sample.

bands characteristic of crystalline $\mathrm{sp}^{2}$ hybridised carbon structures $^{14}$. The sample treated with six pulses is distinct from all others and displays small $\mathrm{D}$ and $\mathrm{D}^{\prime}$ bands indicating a high degree of graphitisation. The $I_{\mathrm{D}} / I_{\mathrm{G}}$ ratio is computed by fitting the bands to a Lorentzian function and measures the structural disorder in the basal plane ${ }^{15}$. Figure $3 \mathrm{~b}$ shows the average from five aleatory locations on each sample. The $I_{\mathrm{D}} / I_{\mathrm{G}}$ ratio is $>1$ for all samples except for the sample treated with six pulses where $I_{\mathrm{D}} / I_{\mathrm{G}}$ is almost zero, indicating a drastic increase of crystalline sizes $\left(L_{a}\right)^{16}$ and a decrease in point defect densities in the basal plane ${ }^{17}$. The $2 \mathrm{D}$ band can be fitted to a single peak for all samples except after six pulses where the band splits into two peaks $\left(2710 \mathrm{~cm}^{-1}\right.$ and $\sim 2737 \mathrm{~cm}^{-1}$ ) as shown in Fig. 3c. This behaviour has been reported as evidence of $3 \mathrm{D}$ ordering by Pimenta et al. ${ }^{18}$ in a study where disordered $\mathrm{sp}^{2}$ carbon films were treated at different temperatures showing the splitting of the $2 \mathrm{D}$ band with increase in stacking order. The splitting observed here supports the XRD data and confirms the efficient conversion of turbostratic into highly crystalline graphite.

TEM confirms that large graphitic crystals dominate the sample after six pulses, accompanied by a small fraction of disordered material (Fig. 4a and Supplementary Fig. 2). The proportions observed are consistent with the XRD analysis of $90 \%$ graphite and $10 \%$ disordered carbon. The graphite regions are easily identified in the TEM due to their straight edges in the TEM images while the disordered portion appears rough. Imaging at higher magnification shows that the disordered regions contain pores and graphenic fringes (Fig. 4b), similar to previous high-resolution TEM images of non-graphitising carbons ${ }^{12,19-21}$. The graphitic crystallites contain the expected fringes and exhibit long-range stacking, consistent with the sharpening of the $\{002\}$ 

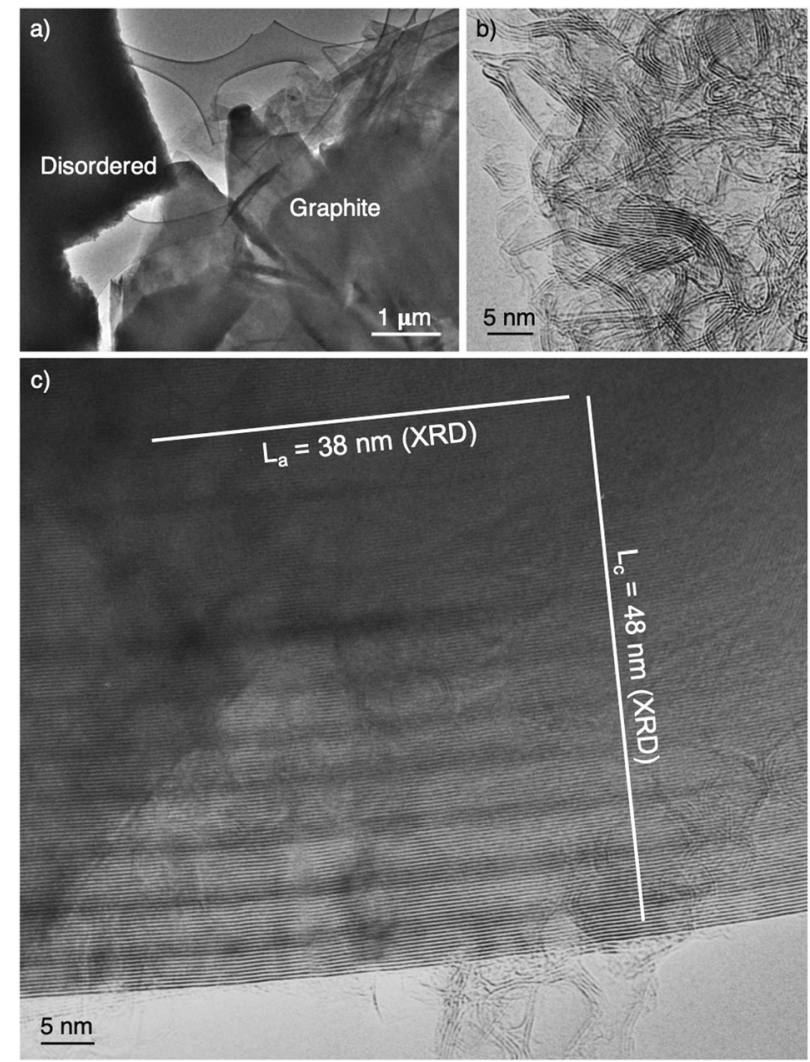

Fig. 4 TEM images of PVDC treated with six high-temperature pulses.

Consistent with the XRD analysis of $90 \%$ graphite, the sample was mostly graphitic, with a small disordered component. a Micrograph showing adjacent disordered and graphite regions. $\mathbf{b}$ HRTEM on the disordered region showing a classic non-graphitising structure. c HRTEM on graphite region in which the crystallite sizes obtained from XRD are superimposed.

reflection and the appearance of $\{101\}$ and $\{112\}$ reflections. XRD also enables determination of the crystalline size via the Scherrer equation. Using a shape factor of unity (see ref. ${ }^{22}$ for a discussion), analysis of the $\{100\}$ and $\{002\}$ reflections yields $L_{a}=38 \mathrm{~nm}$ and $L_{c}=48 \mathrm{~nm}$, respectively. These values are overlaid as arrows in Fig. $4 \mathrm{c}$ and are consistent with the TEM image. We also performed TEM after five pulses and observed an abundance of disordered regions with few graphitic regions; this is consistent with the XRD intensity ratios, which found a $90 \%$ disordered component.

In order to show that PVDC is not an isolated case, we applied the same methodology to cellulose $\left(\mathrm{C}_{6} \mathrm{H}_{10} \mathrm{O}_{5}\right)$, another wellknown non-graphitising carbon ${ }^{5,12,19}$. Cellulose also underwent graphitisation using the AAS, albeit more slowly than PVDC. After six pulses, the $\{002\}$ reflection shows a broad disordered peak with a small sharp peak associated with turbostratic graphenic carbon (Fig. 5a). With further pulsing, two distinct peaks develop, indicating that graphite is also present. The $L_{c}$ value associated with the graphite component is $32 \mathrm{~nm}$, comparable to that seen for PVDC. After 30 pulses, the $\{100\}$ and $\{101\}$ peaks can be observed (Fig. 5b) indicating 3D ordering. While a broad $\{10\}$ peak remains, the sample comprises around $50 \%$ ordered material $(\mathrm{T}+\mathrm{G})$, at least ten times higher than typical observations where the degree of ordered material is at best a few percent. Even though the process is slower in cellulose, the trajectory towards a complete transformation is clear. The similarity between PVDC and cellulose can be seen in the TEM images of graphitised (Fig. 5d) and non-graphitised (Fig. 5e) regions of cellulose, which closely resemble their PVDC counterparts.
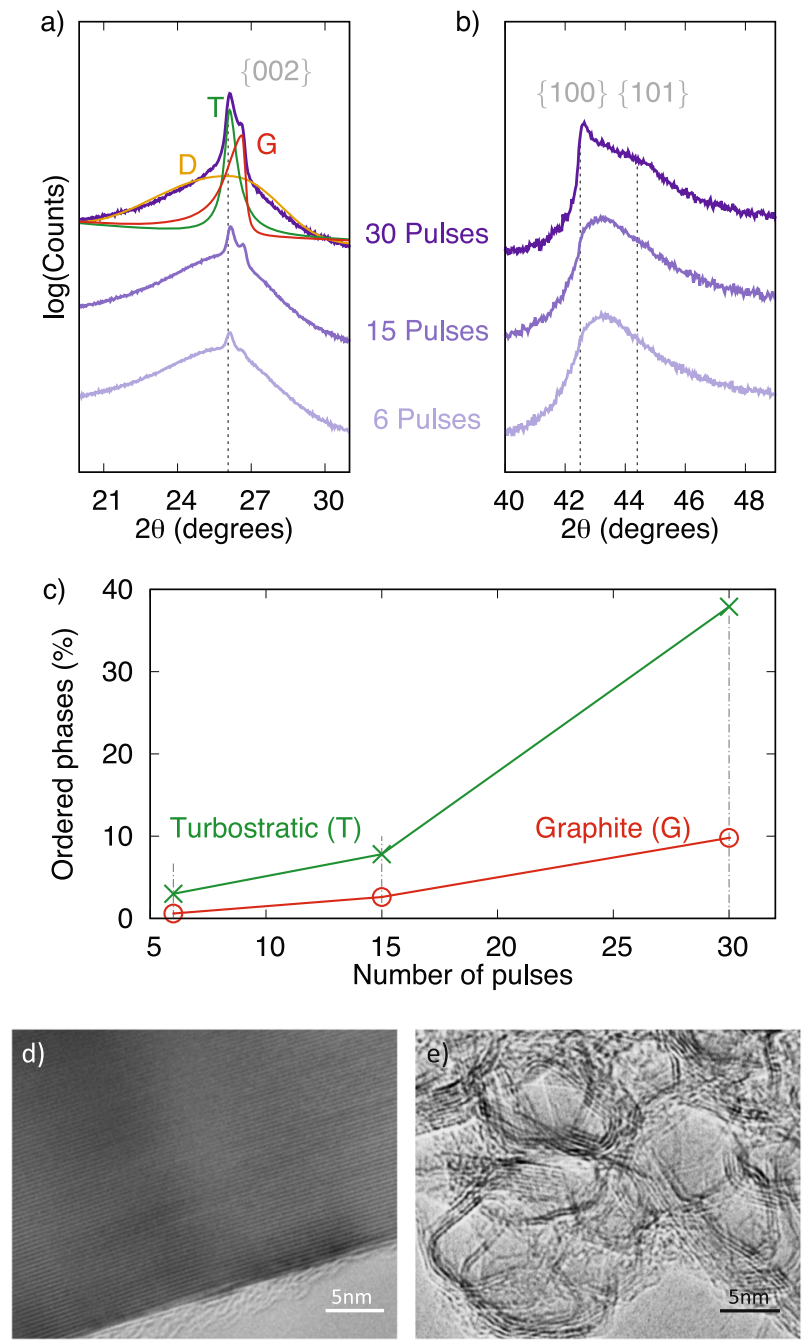

Fig. 5 Analysis of cellulose treated with high-temperature pulses. a Magnification of the $\{002\}$ reflection with the 30 -pulse sample showing the disordered $(D)$, turbostratic $(T)$ and graphitic $(G)$ components. b Magnification of the $\{100\}$ and $\{101\}$ reflections with the 30-pulse sample showing the respective components. c Percentage of turbostratic and graphite phases as computed from the intensity ratios of the $\{002\}$ reflection. d HRTEM image of a graphitised region showing the stacked graphitic fringes. e HRTEM image of a classic non-graphitising region.

Raman spectroscopy of the cellulose samples further confirms the trajectory towards graphitic order; see Supplementary Fig. 3.

\section{Discussion}

A critical question to answer is why our method enables graphitisation of a non-graphitising carbon, while a conventional furnace does not. Our process differs in two ways: (i) the repeated fast-pulsed nature of heating, and (ii) the rapid speed at which $3000^{\circ} \mathrm{C}$ is achieved (several seconds versus many hours in conventional furnaces). We propose that oxygen (in the form of $\mathrm{O}_{2}$ and moisture) is adsorbed between pulses and chemically attacks non-hexagonal defects during the subsequent pulse. This idea is consistent with studies of carbon nanohorns and nanotubes where oxidation occurs preferentially at pentagonal rings ${ }^{23-25}$ and is exploited to access internal cavities by etching the tips ${ }^{26-28}$. Experimental and theoretical works ${ }^{29-34}$ have shown that defects such as pentagons, heptagons and octagons are arranged in lines, forming extended defects. An example of a model of a porous carbon showing these extended defects is depicted in Fig. $6 a^{32}$. 

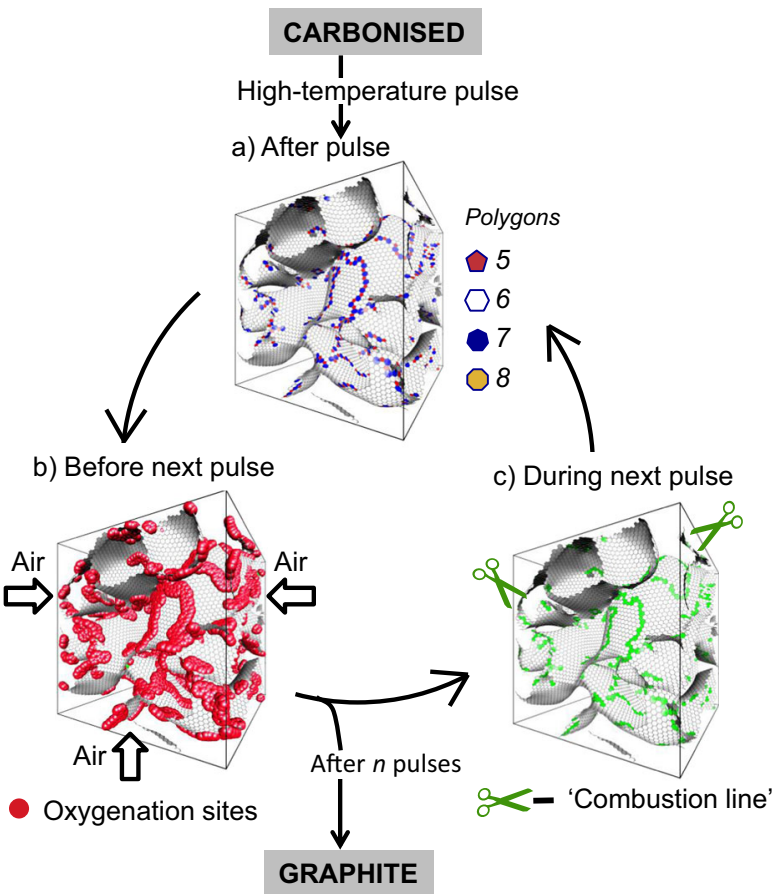

d) Oxygen content (\%)
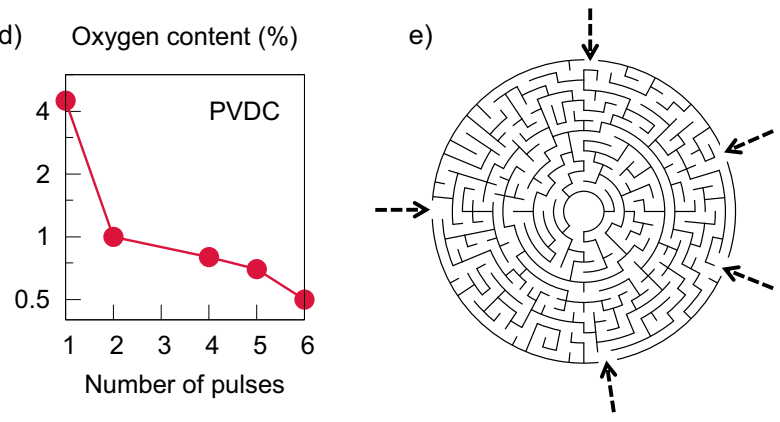

Fig. 6 Proposed mechanism for graphitisation by high-temperature pulsing. a After a given pulse, the structure contains regions of hexagonal bonding separated by linear chains of pentagons, heptagons and octagons. b Between pulses, atmospheric oxygen is adsorbed within the structure. c During the subsequent pulse, trapped oxygen preferentially etches the linear chains marked as "combustion lines". The atomistic model is from refs. ${ }^{32,43}$. d Oxygen content determined from XPS of samples of PVDC after different number of pulses. Full O1s and C1s spectra can be found in Supplementary Fig. 4. e Maze-like model of a non-graphitising carbon based on diagrams from ref. ${ }^{12}$

The extended lines of non-hexagonal carbon are the basis for a thought experiment to explain the unexpected graphitisation seen here. When oxygen gets adsorbed into the network of the nongraphitising carbon, it will preferentially attach along these lines, either by physisorption or chemisorption (Fig. 6b). Upon rapid heating, the combustion temperature is exceeded before the oxygen can escape through the nanoporous network (Fig. 6e). Combustion of carbon in the pentagons and heptagons acts like scissors (Fig. 6c), cutting the structure into independent graphene flakes. With an increasing number of pulses, the network starts to collapse, allowing the flakes to progressively stack into graphite. Conceptually, this approach resembles a percolation model, in which the structure suddenly rearranges in a manner akin to the collapse of the wooden blocks in Jenga. This model is consistent with our PVDC data that shows an increase in graphitic ordering up to five pulses, followed by a sudden change upon the sixth pulse.
The progressive reduction in "combustion lines" is supported by X-ray photoelectron spectroscopy (Fig. 6d). After one pulse, the sample is similar to non-graphitising carbons from a conventional furnace and contains the maximum amount of oxygen as it has the greatest porosity. Over subsequent pulses, the oxygen content continues to gradually decrease. Note that the oxygen content does not have a 1:1 relationship with the fraction of ordered phases (Fig. 2d) because widespread graphitic order does not appear until the final critical defect lines are combusted.

This combustion route to graphite unifies past literature models of graphitisation, which have been the subject of ongoing debate. While pentagons ${ }^{35}$ and heptagons ${ }^{36}$ have both been proposed as sources of positive and negative curvature, respectively, a key aspect of our mechanism is their arrangement in lines as proposed more recently ${ }^{29-34}$. The pentagon/heptagon chains can be understood as the (unspecified) cross-links proposed by Franklin, which locked nanocrystallites together ${ }^{3}$, while collapse of the structure is reminiscent of the "falling cards" model of Dahn et al. ${ }^{37}$. The cutting of the structure develops hightemperature BSUs that resemble the conventional carbonisation BSUs as proposed by Oberlin et al. ${ }^{38}$, while an important difference is that the very high temperature of $3000^{\circ} \mathrm{C}$ gives the fragments sufficient energy (around $9 \mathrm{eV} /$ atom; see Supplementary Fig. 5) to rearrange into graphitic stacks.

Inherent to the combustion route is the nanoporosity of the carbon network, which is often likened to a maze model ${ }^{12}$ as shown schematically in Fig. 6e. Once air has been adsorbed, the tortuous path between the internal voids and the external environment makes escape difficult, especially if the change in temperature is rapid. The role played by oxygen in our work resembles a study by Burket et al. ${ }^{39}$ who observed partial graphitisation when activated carbon was heated to $2000^{\circ} \mathrm{C}$. Activated carbons are formed by oxidising a non-graphitising carbon, and we suggest that their result can be explained by oxygen selectively removing the structural units that inhibit graphitisation.

In this work, we use a common piece of equipment from chemistry laboratories to obtain graphite from two polymers that are universally considered to be non-graphitising. We propose that this occurs due to combustion of defect lines via adsorbed atmospheric oxygen. Further experiments that restrict adsorbed oxygen are required to fully confirm the proposed mechanism, but these are beyond the capabilities of our off-the-shelf AAS. Our observations question the traditional dichotomy between graphitising and non-graphitising carbons that have framed discussion for nearly 70 years and support the recent theory of Ouzilleau et al. that all carbons graphitise to some extent ${ }^{40}$. Our results take their idea even further, showing that PVDC, a classic non-graphitising carbon, can be transformed almost entirely into graphite. Measurements on cellulose, another classic nongraphitising carbon, demonstrate that PVDC is not a unique case. While the transformation of cellulose is slower, it follows a similar trajectory. It is noteworthy that the vast majority of carbonaceous materials are generally considered to fall under the non-graphitising umbrella and have not previously been considered as a source of graphite. Harvesting these materials via the combustion route to graphite opens up a route for manufacturing high-quality graphite from abundant and low-cost sources such as biomass. The challenge now is to explore and scale-up this process.

During the review process, we became aware of an interesting study by Luong et al. ${ }^{41}$ who reported flash Joule heating of nongraphitising materials. The resultant material contained a modest number of graphene layers with a turbostratic spacing. This material, which they called flash graphene, is quite different to our samples that contain extended graphite crystals with the Bernal spacing. Despite these significant differences, both works 
direct attention to the merits of using pulsed heating to transform non-graphitising carbons into ordered carbon.

\section{Methods}

Temperature treatments. Samples are heated in an inert atmosphere inside an STF 1200 tube furnace over $4 \mathrm{~h}$ to a carbonisation temperature of $1000^{\circ} \mathrm{C}$. This temperature is held for a further hour before the sample is cooled passively back to room temperature. The resulting carbon sample is then milled in a mortar and pestle before high-temperature pulsing in an Agilent GTA120 AAS. The temperature profile of each AAS pulse is as follows: room temperature- $100^{\circ} \mathrm{C}$ in $5 \mathrm{~s}$, $100-3000^{\circ} \mathrm{C}$ in $15 \mathrm{~s}$, holding at $3000^{\circ} \mathrm{C}$ for a further $15 \mathrm{~s}$ before cooling from 3000 to $200^{\circ} \mathrm{C}$ in $13 \mathrm{~s}$. Argon flow is maintained during the AAS pulsing. While the AAS is used here in an innovative way, this equipment is not designed for long pulses and the parameters used here are the upper limit before the thermal cutout triggers.

The use of an AAS for high-temperature treatment brings certain limitations, arising from the fact that the instrument is first and foremost a spectrometer, and not a general-purpose high-temperature furnace. The most notable limitations are restrictions on sample size and the inability to maintain elevated temperatures for extended times. The physical dimensions of the graphite tube furnace in the AAS preclude the treatment of samples larger than circa $50 \mathrm{mg}$. Second, while the AAS is capable of repeatingly achieving temperatures of $3000^{\circ} \mathrm{C}$, the cooling system is not designed to maintain this temperature for $>30 \mathrm{~s}$ or so. For this reason, the furnace is allowed to cool for a few minutes between pulses.

Characterisation techniques. XRD is performed in a Bruker D8 Advance Diffractometer with Bragg-Brentano geometry and a $\mathrm{Cu} \mathrm{K}-\alpha$ source. XRD specimens are prepared by placing a powder sample onto a low signal silicon wafer holder. Patterns are collected over a $2 \theta$ range of $10-90^{\circ}$ with a dwell time of $1.5 \mathrm{~s}$ and step size of $0.03^{\circ}$. Further information regarding the device configuration can be found in ref. ${ }^{22}$.

Raman spectra is measured using a Renishaw inVia Raman microscope with $488 \mathrm{~nm}$ excitation. The powder samples are directly deposited onto a glass slide.

TEM is performed using two instruments, a JEOL 2010 and a TALOS FS200X, using an accelerated voltage of $200 \mathrm{keV}$ and current density typically of $15 \mathrm{pA} / \mathrm{cm}^{2}$. Care is taken to minimise sample time under the beam $(<2 \mathrm{~min})$ to avoid beam damage. The samples are prepared by grinding in an agate mortar in methanol followed by sonication before depositing on lacey carbon grids.

$\mathrm{X}$-ray photoelectron spectroscopic measurements are performed using a Physical Electronics VersaProbe II instrument equipped with a monochromatic Al $\mathrm{K}-\alpha \mathrm{X}$-ray source $(\mathrm{h} v=1486.7 \mathrm{eV})$ and a concentric hemispherical analyser. Charge neutralisation is performed using both low-energy electrons $(<5 \mathrm{eV})$ and argon ions. The binding energy axis is calibrated using sputter cleaned $\mathrm{Cu}(\mathrm{Cu} 2 p 3 /$ $2=932.62 \mathrm{eV}, \mathrm{Cu} 3 p 3 / 2=75.1 \mathrm{eV})$ and Au foils $(\mathrm{Au} 4 f 7 / 2=83.96 \mathrm{eV})^{42}$. Peaks are referenced to $\mathrm{CH}_{x}$ band in the carbon 1s spectra at $284.8 \mathrm{eV}$. Measurements are made at a takeoff angle of $45^{\circ}$ with respect to the sample surface plane resulting in a typical sampling depth of 3-6 nm (95\% of the signal originated from this depth or shallower). Quantification is done using instrumental relative sensitivity factors that account for the X-ray cross-section and inelastic mean free path of the electrons.

\section{Data availability}

The data generated and analysed during the current study are available from the authors on reasonable request.

Received: 28 January 2020; Accepted: 4 June 2020;

Published online: 24 July 2020

\section{References}

1. Jara, A. D., Betemariam, A., Woldetinsae, G. \& Kim, J. Y. Purification, application and current market trend of natural graphite: a review. Int. J. Mining Sci. Technol. 29, 671-689 (2019).

2. Pierson, H. O. Activated Carbon 1st edn (William Andrew Publishing, 1993).

3. Franklin, R. E. Crystallite growth in graphitizing and non-graphitizing carbons. Proc. R. Soc. A Math. Phys. Eng. Sci. 209, 196-218 (1951).

4. Fitzer, E., Kochling, K. H., Boehm, H. P. \& Marsh, H. Recommended terminology for the description of carbon as a solid - (IUPAC recommendations 1995). Pure Appl. Chem. 67, 473-506 (1995).

5. Kobayashi, K., Sugawara, S., Toyoda, S. \& Honda, H. An X-ray diffraction study of phenol-formaldehyde resin carbons. Carbon 6, 359-363 (1968).

6. Oberlin, A. \& Rousseaux, F. Graphitation partielle de quelques carbones durs. Etude en microscopie et microdiffraction électroniques. J. Appl. Crystallogr. 1, 218-226 (1968).

7. Kamiya, K. \& Suzuki, K. Preferential alignment of carbon layers around pores in hard carbon and multi-phase graphitization. Carbon 13, 317-320 (1975).
8. Kasahara, N., Shiraishi, S. \& Oya, A. Heterogeneous graphitization of thin carbon fiber derived from phenol-formaldehyde resin [4]. Carbon 41, 1654-1656 (2003).

9. Oya, A. \& Marsh, H. Phenomena of catalytic graphitization. J. Mater. Sci. 17, 309-322 (1982)

10. Oya, A. \& Otani, S. Catalytic graphitization of carbons by various metals. Carbon 17, 131-137 (1979)

11. Krivoruchko, O. P., Shmakov, A. N. \& Zaikovskii, V. I. In situ X-ray diffraction study of solid state transformations during catalytic graphitisation of amorphous carbon. Nucl. Instrum. Methods Phys. Res. A 470, 198-201 (2001).

12. Marsh, H. \& Rodríguez-Reinoso, F. Activated Carbon 1st edn (Elsevier Science \& Technology, 2006).

13. Bernal, J. D. The structure of graphite. Proc. R. Soc. A Math. Phys. Eng. Sci. 106, 749-773 (1924).

14. Ferrari, A. C. \& Basko, D. M. Raman spectroscopy as a versatile tool for studying the properties of graphene. Nat. Nanotechnol. 8, 235-246 (2013).

15. Ferrari, A. C. Raman spectroscopy of graphene and graphite: disorder, electron-phonon coupling, doping and nonadiabatic effects. Solid State Commun. 143, 47-57 (2007).

16. Ribeiro-Soares, J. et al. Structural analysis of polycrystalline graphene systems by Raman spectroscopy. Carbon 95, 646-652 (2015).

17. Lucchese, M. M. et al. Quantifying ion-induced defects and Raman relaxation length in graphene. Carbon 48, 1592-1597 (2010).

18. Pimenta, M. A. et al. Studying disorder in graphite-based systems by Raman spectroscopy. Phys. Chem. Chem. Phys. 9, 1276-1291 (2007).

19. Harris, P. Structure of non-graphitising carbons. Int. Mater. Rev. 42, 206-218 (1997).

20. Ban, L. L., Crawford, D. \& Marsh, H. Lattice-resolution electron-microscopy in structural studies of non-graphitizing carbons from polyvinylidene chloride (PVDC). J. Appl. Crystallogr. 8, 415-420 (1975).

21. Harris, P. \& Tsang, S. C. High-resolution electron microscopy studies of nongraphitizing carbons. Philos. Mag. A 76, 667-677 (1997)

22. Putman, K. J. et al. Pulsed thermal treatment of carbon up to $3000^{\circ} \mathrm{C}$ using an atomic absorption spectrometer. Carbon 135, 157-163 (2018).

23. Ajayan, P. M. et al. Opening carbon nanotubes with oxygen and implications for filling. Nature 362, 522-525 (1993).

24. Van Lier, G., Ewels, C. P., Cases-Amat, M., Suarez-Martinez, I. \& Geerlings, P. Comparison between early stage oxygenation behavior of fullerenes and carbon nanotubes. J. Nanosci. Nanotechnol. 9, 6113-6119 (2009).

25. Tsang, S. C., Harris, P. J. \& Green, M. L. Thinning and opening of carbon nanotubes by oxidation using carbon dioxide. Nature 362, 520-522 (1993).

26. Utsumi, S. et al. Opening mechanism of internal nanoporosity of single-wall carbon nanohorn. J. Phys. Chem. B 109, 14319-14324 (2005).

27. Karousis, N., Suarez-Martinez, I., Ewels, C. P. \& Tagmatarchis, N. Structure, properties, functionalization, and applications of carbon nanohorns. Chem. Rev. 116, 4850-4883 (2016).

28. Ugarte, D., Chatelain, A. \& de Heer, W. A. Nanocapillarity and chemistry in carbon nanotubes. Science 274, 1897-1899 (1996).

29. Farbos, B. et al. Nanoscale structure and texture of highly anisotropic pyrocarbons revisited with transmission electron microscopy, image processing, neutron diffraction and atomistic modeling. Carbon 80, 472-489 (2014).

30. Gamboa, A., Farbos, B., Aurel, P., Vignoles, G. L. \& Leyssale, J.-M. Mechanism of strength reduction along the graphenization pathway. Sci. Adv. 1, e1501009 (2015).

31. Leyssale, J.-M., Da Costa, J.-P., Germain, C., Weisbecker, P. \& Vignoles, G. Structural features of pyrocarbon atomistic models constructed from transmission electron microscopy images. Carbon 50, 4388-4400 (2012).

32. Martin, J. W., de Tomas, C., Suarez-Martinez, I., Kraft, M. \& Marks, N. A. Topology of disordered 3D graphene networks. Phys. Rev. Lett. 123, 116105 (2019).

33. Guo, J. et al. Topological defects: origin of nanopores and enhanced adsorption performance in nanoporous carbon. Small 8, 3283-3288 (2012).

34. Huang, P. Y. et al. Grains and grain boundaries in single-layer graphene atomic patchwork quilts. Nature 469, 389-392 (2011).

35. Harris, P. J. F. New perspectives on the structure of graphitic carbons. Crit. Rev. Solid State Mater. Sci. 30, 235-253 (2005).

36. Terrones, H. \& Mackay, A. L. Negatively curved graphite and triply periodic minimal-surfaces. J. Math. Chem. 15, 183-195 (1994).

37. Dahn, J., Xin, W. \& Gao, Y. The "falling cards model" for the structure of microporous carbons. Carbon 35, 825-830 (1997).

38. Oberlin, A. Carbonization and graphitization. Carbon 22, 521-541 (1984).

39. Burket, C. L., Rajagopalan, R. \& Foley, H. C. Overcoming the barrier to graphitization in a polymer-derived nanoporous carbon. Carbon 46, 501-510 (2008).

40. Ouzilleau, P., Gheribi, A. E., Chartrand, P., Soucy, G. \& Monthioux, M. Why some carbons may or may not graphitize? The point of view of thermodynamics. Carbon 149, 419-435 (2019). 
41. Luong, D. X. et al. Gram-scale bottom-up flash graphene synthesis. Nature 577, 647-651 (2020)

42. Seah, M. P. Summary of ISO/TC 201 standard: VII ISO $15472: 2001$ - surface chemical analysis - X-ray photoelectron spectrometers - calibration of energy scales. Surf. Interface Anal. 31, 721-723 (2001).

43. de Tomas, C., Suarez-Martinez, I. \& Marks, N. A. Carbide-derived carbons for dense and tunable 3D graphene networks. Appl. Phys. Lett. 112, 251907 (2018).

\section{Acknowledgements}

The authors thank Oscar Del Borrello from the University of Western Australia for his assistance in the use of the Atomic Absorption Spectrometer. Part of the research was undertaken using the XRD and TEM instrumentation at the John de Laeter Centre, Curtin University.

\section{Author contributions}

J.L.F. and K.J.P. performed the high-temperature experiments and XRD characterisation. J.L.F. and P.J.F.H. performed microscopic characterisation. T.Z., Y.L. and M.T. performed the Raman and XPS characterisation. J.L.F., N.A.M. and I.S.-M. designed the experiments and wrote the manuscript.

\section{Competing interests}

The authors declare no competing interests.

\section{Additional information}

Supplementary information is available for this paper at https://doi.org/10.1038/s43246020-0045-y.

Correspondence and requests for materials should be addressed to I.S.-M.

Reprints and permission information is available at http://www.nature.com/reprints

Publisher's note Springer Nature remains neutral with regard to jurisdictional claims in published maps and institutional affiliations.

\begin{abstract}
cc (i) Open Access This article is licensed under a Creative Commons Attribution 4.0 International License, which permits use, sharing, adaptation, distribution and reproduction in any medium or format, as long as you give appropriate credit to the original author(s) and the source, provide a link to the Creative Commons license, and indicate if changes were made. The images or other third party material in this article are included in the article's Creative Commons license, unless indicated otherwise in a credit line to the material. If material is not included in the article's Creative Commons license and your intended use is not permitted by statutory regulation or exceeds the permitted use, you will need to obtain permission directly from the copyright holder. To view a copy of this license, visit http://creativecommons.org/ licenses/by/4.0/.
\end{abstract}

(C) The Author(s) 2020 